\title{
How to analyze transcriptome of each of 800000 cells, using automatized library prep for Illumina from $10 \times$ Genomics (technology overview)
}

\author{
K. Bolaeva \\ SkyGen LLC \\ e-mail: kermenbolaeva@gmail.com
}

Key words: transcriptome of single cells, UMI, unique molecular barcodes, gel bead technology, RNA-seq on Illumina platform, microfluidics

Motivation and Aim: Nowadays plenty of new approaches for library prep for Illumina sequencing are developed every day. As well, scientists are more and more interested, what is going on in a different types of tissue or in a tumor microenvironment. So, in this technology review we are to describe a protocol from $10 \times$ Genomics automated library preparation, which enables to mark cDNA from 80000 single cells using microfluidics, gel beads coated with millions of oligos and unique molecular barcoding technology (UMI). Currently scientists know that in a tissue there are different types of cells, each of them has it's own function. Great example for that is a brain tissue. It was shown, that $10 \times$ Genomics approach got it possible to analyze more than 1 million of cells of a rat brain, and divide all cells into different types based on their expression profile. As well, in a tumor tissue we also have healthy cells, tumor cells and it could be interesting to see and quantify ratio of them. Another amazing example of the technology application is investigation of blood cell, and especially of peripheral blood mononuclear cells (PBMC). PBMC cells were divided by different types of gene expression, and therefore different Methods and Algorithms: One of the main advantages of the technology discussed here is, that due to automatization using Chromium Controller from $10 \times$ Genomics, we can mark cDNA from 800000 cells in 7 minutes, allowing to distinguish transcriptome data from every singe cell finally. It is possible to analyze thousands of single cells in every run. $10 \times$ technology suite performs millions of parallel reactions to enable gene expression profiling at scale with single cell resolution. It is crucial to have suspension of alive eukaryotic cells for the experiment. Then suspension of cells, thousands gel beads coated with millions of oligos (oligos from each gel bead are unique, as well as oligos within one gel bead are with UMI), and oil are put into a microfluidic chip of Chromium Controller machine. Cells and beads under pressure are migrating in a microfluidic channels, where they are mixed with oil so, that finally re get drops in the oil. In each drop we expect to have one gel bead and one cell. Cell is to lysates within the drop, and mRNA molecules are attaching to poly(T) part of oligos immobilized on the surface of gel beads. After that, gel beads are also dissolved. After reverse transcription reaction, molecules of cDNA has on their ends UMI, 10× Genomics barcode, and Illumina P5 and P7 primers. Ready cDNA can then be estimated, and then used for the Illumina library prep. Finally it was described that around 20000 reads can be obtained per cell.

\section{References}

1. Wang J. et al. (2018) Gene expression distribution deconvolution in single-cell RNA sequencing. Proc Natl Acad Sci USA.

2. Lambrechts D. et al. (2018) Phenotype molding of stromal cells in the lung tumor microenvironment. Nat Med.

3. Griffiths J.A. et al. (2018) Detection and removal of barcode swapping in single-cell RNA-seq data. Nat Commun. 